\title{
MOSCO CONVERGENCE AND THE KADEC PROPERTY
}

\author{
JONATHAN M. BORWEIN AND SIMON FITZPATRICK
}

(Communicated by William J. Davis)

\begin{abstract}
We study the relationship between Wijsman convergence and Mosco convergence for sequences of convex sets. Our central result is that Mosco convergence and Wijsman convergence coincide for sequences of convex sets if and only if the underlying space is reflexive with the dual norm having the Kadec property.
\end{abstract}

\section{INTRODUCTION}

There are many notions of convergence of sequences of convex sets in a normed space. This paper was stimulated by the analysis in Beer [Be] where various notions of set convergence are characterized when the sets in question are hyperplanes. One of the most fruitful notions is that of Mosco convergence [At, Be, Mo1, Mo2, Sa-We, So, Ts]. Mosco convergence is particularly useful in reflexive spaces since then polarity is sequentially bicontinuous $\left(E_{n}\right.$ converges to $E$ if and only if $E_{n}^{0}$ converges to $E^{0}$; see [Mo2]). Another more intuitive notion is that of Wijsman convergence [Wi]. It has been known for some time that these notions coincide when the underlying space is reflexive and the norm used is Fréchet differentiable [At, So, Ts] and when the space is finite dimensional. Beer has shown that this coincidence fails, even for sequences of hyperplanes, whenever the dual norm is not Kadec.

Our central result (Theorem 3.4) is that Mosco convergence and Wijsman convergence coincide for sequences of convex sets if and only if the underlying space is reflexive with the dual norm having the Kadec property. This recaptures the results mentioned above. We establish this by providing a circuit of characterizations of reflexive spaces whose dual norm is Kadec (Theorem 3.1). The proof of this result is motivated both by Tsukada's technique and Beer's analysis. In $\S 2$ we make the appropriate definitions and provide some preliminary results.

Received by the editors August 22, 1988, and in revised form November 15, 1988.

1980 Mathematics Subject Classification (1985 Revision). Primary 54C60, 46B20.

The first author was supported in part by NSERC. 


\section{Preliminary Results}

Throughout this paper $X$ is a real Banach space endowed with a fixed norm, \|\| .

A sequence of sets $\left\{C_{n}\right\}$ in $X$ is said to converge Wijsman to a set $C$ if

$$
\lim _{n \rightarrow \infty} d\left(x, C_{n}\right)=d(x, C)
$$

for each $x$ in $X$, where $d(x, C):=\inf _{c \in C}\|x-c\|$ and $d(x, \varnothing)=\infty$. We will write $\lim _{W} C_{n}=C$.

A sequence of sets $\left\{C_{n}\right\}$ in $X$ is said to converge Mosco to a set $C$ if

$\mathrm{M}(\mathrm{i})$ : for each $x \in C$ there exist, for large $n, x_{n} \in C_{n}$ such that $x_{n}$ converges in norm to $x$.

M(ii): if there is a subsequence $n^{\prime}$ with $x_{n^{\prime}} \in C_{n^{\prime}}$ such that $x_{n^{\prime}}$ converges weakly to a point $x$ then $x \in C$.

We will write $\lim _{M} C_{n}=C$.

It is apparent that Wijsman convergence depends on the precise norm used, while Mosco convergence is preserved by equivalent renorming. We now record some useful relationships.

Proposition 2.1. (a) If $C_{n}$ are subsets of $X$ and $\lim _{W} C_{n}=\varnothing$ then $\lim _{M} C_{n}=\varnothing$.

(b) Conversely, if $X$ is reflexive and $\lim _{M} C_{n}=\varnothing$ then $\lim _{W} C_{n}=\varnothing$.

Proof. (a) For $C$ empty, $\mathbf{M}(\mathrm{i})$ holds vacuously. If $\mathrm{M}$ (ii) fails with $C$ empty then there is a subsequence $n^{\prime}$ with $x_{n^{\prime}} \in C_{n^{\prime}}$ such that $x_{n^{\prime}}$ converges weakly to a point $x$. But now $\left\{x_{n^{\prime}}\right\}$ is bounded and $\liminf d\left(x, C_{n}\right)<\infty$ whence $\lim _{W} C_{n}=\varnothing$.

(b) If $\liminf d\left(x, C_{n}\right)<\infty$ we can find a bounded subsequence $c_{n^{\prime}} \in C_{n^{\prime}}$. By weak compactness there is a further subsequence $c_{n^{\prime \prime}} \in C_{n^{\prime \prime}}$ with weak limit $x_{0}$. By $\mathbf{M}$ (ii) we see that $x_{0} \in \lim _{M} C_{n}=\varnothing$. This contradiction establishes that $\liminf d\left(x, C_{n}\right)=\infty$ so that $\lim _{W} C_{n}=\varnothing$.

Theorem 2.2. (a) If $X$ is reflexive and $\lim _{M} C_{n}=C$ then $\lim _{W} C_{n}=C$.

(b) If $X$ is nonreflexive there is a sequence $\left\{C_{n}\right\}$ of compact convex sets with $\lim _{M} C_{n}=C$ compact convex and nonempty, but $\lim _{W} C_{n}$ fails to exist.

Proof. (a) We may suppose that $C$ is nonempty. Let $\varepsilon>0$. Choose $c \in C$ so that $\|x-c\| \leq d(x, C)+\varepsilon$. By $\mathrm{M}(\mathrm{i})$ select $c_{n} \in C_{n}$ with $c_{n}$ converging in norm to $c$. Then

$$
\lim \sup d\left(x, C_{n}\right) \leq \lim \left\|x-c_{n}\right\|=\|x-c\| \leq d(x, C)+\varepsilon,
$$

and so

$$
\lim \sup d\left(x, C_{n}\right) \leq d(x, C) .
$$

Suppose now that $\liminf d\left(x, C_{n}\right)<d(x, C)-\varepsilon$. Select $c_{n^{\prime}} \in C_{n^{\prime}}$ with $\left\|x-c_{n^{\prime}}\right\|<d(x, C)-\varepsilon$. Since $X$ is reflexive $\left\{c_{n^{\prime}}\right\}$ has a weak subsequential 
limit point $x_{0}$ which, by $\mathrm{M}(\mathrm{ii})$, lies in $C$. Thus, as the norm is weakly lower semicontinuous

$$
d(x, C) \leq\left\|x-x_{0}\right\| \leq d(x, C)-\varepsilon,
$$

a contradiction.

(b) Suppose $X$ is not reflexive. Select $e$ in $X$ with $\|e\|=1$. Consider $K:=B[0,1] \cap B\left[e, \frac{1}{2}\right]$. (Here $B[x, r]$ is the closed ball of radius $r$ around $x$.) Since $K$ contains $B\left[\frac{3}{4} e, \frac{1}{4}\right], K$ is not countably weakly compact and we can find $e_{n}$ with $\left\|e_{n}\right\| \leq 1,\left\|e-e_{n}\right\| \leq \frac{1}{2}$ so that $\left\{e_{n}\right\}$ has no weak cluster point [Da]. We now let $C_{n}:=$ ordinary $\left\{0, e_{n}\right\}$ and $C:=\{0\}$.

We first establish that $\lim _{M} C_{n}=C$. Since $0 \in C_{n}$ for all $n$, it suffices to show that if $0 \leq t_{n^{\prime}} \leq 1$ and $t_{n^{\prime}} e_{n^{\prime}}$ converges weakly to $x$ then $x=0$. We may assume, on extracting a further subsequence, that $t_{n^{\prime}}$ converges to $t$. If $t=0$ then $x=0$ since $\left\|e_{n}\right\| \leq 1$. If $t>0$ then $e_{n^{\prime}}$ converges weakly to $t^{-1} x$, which is impossible.

Suppose that $\lim _{W} C_{n}=C^{\prime}$. For any $y \in C^{\prime}$ we have $d\left(y, C_{n}\right) \rightarrow 0$. By M(ii) we see that $y=0$. Thus $C^{\prime}=C=\{0\}$. However,

$$
d\left(e, C_{n}\right) \leq\left\|e_{n}-e\right\| \leq \frac{1}{2}
$$

while $d(e, C)=\|e\|=1$ and $\lim _{W} C_{n} \neq C$.

Part (a) of the previous result is well known (see [So], p. II.6).

Corollary 2.3. A Banach space $X$ is reflexive if and only if Mosco convergence implies Wijsman convergence for sequences of closed convex subsets of $X$.

This corollary explains the need for reflexivity in our main results. The next result similarly explains the need for convexity of the subsets.

Theorem 2.4. A Banach space $X$ is finite dimensional if and only if for every sequence $\left\{C_{n}\right\}$ of weakly closed subsets of $X$, and weakly closed set $C$

$$
\lim _{W} C_{n}=C \text { if and only if } \lim _{M} C_{n}=C .
$$

Proof. First, suppose $X$ is finite dimensional. Then Wijsman and Mosco convergences coincide for closed sets [Au, p. 244]. Second, Theorem 2.2(b) covers the nonreflexive case.

Suppose now that $X$ is reflexive and infinite dimensional. Since 0 is in the weak closure of the unit sphere, we can choose a sequence $\left\{e_{n}\right\}$ of unit vectors converging weakly to zero (by the Kaplansky-Whitley construction, [Da, p. 58]). Select a norm-one linear functional $f$, and define

$$
C:=\left\{x: \mid f(x) \geq \frac{1}{2}\right\} \text { and } C_{n}:=C \cup\left\{e_{n}\right\} .
$$

An easy computation shows that

$$
d(x, C)=\max \left\{0, \frac{1}{2}-|f(x)|\right\}
$$

and

$$
d\left(x, C_{n}\right)=\min \left\{d(x, C),\left\|x-e_{n}\right\|\right\} \leq d(x, C) .
$$


Observe that $\liminf \left\|x-e_{n}\right\| \geq \max \{\|x\|, 1-\|x\|\} \geq \frac{1}{2} \geq d(x, C)$ for all $x$ (because $\left\{e_{n}\right\}$ converges weakly to zero and the norm is weakly lower semicontinuous). This shows that $\liminf d\left(x, C_{n}\right) \geq d(x, C)$ and so $\lim _{W} C_{n}=C$. Also $C$ and each $C_{n}$ is weakly closed. However, $e_{n} \in C_{n}$ and $\left\{e_{n}\right\}$ converges weakly to $0 \notin C$. Thus $\lim _{M} C_{n} \neq C$.

Remark 2.5. A sequence $\left\{C_{n}\right\}$ of subsets of $X$ converges Mosco [Wijsman] to a set $C$ if and only if $\left\{\mathrm{cl} C_{n}\right\}$ converges Mosco [Wijsman] to $C$. Thus there is no loss of generality in considering only norm-closed sets.

\section{Dual Kadec SPaces}

Three definitions are needed. First recall that the duality map $J$ between $X$ and $X^{*}$ is the subgradient of $\frac{1}{2}\|\|^{2}$; explicitly $x^{*} \in J(x)$ if and only if $\left\|x^{*}\right\|^{2}=\|x\|^{2}=\left\langle x^{*}, x\right\rangle$. Also $J$ is said to norm-usco provided that $J$ is norm to norm upper semicontinuous with norm compact images (see [GGS]). In particular, if either the norm is Fréchet or $X$ is finite dimensional then $J$ is norm-usco. A dual Banach space $X^{*}$ is (sequentially) weak ${ }^{*}$ Kadec if whenever $\left\{x_{n}^{*}\right\}$ is a sequence of norm-one elements of $X^{*}$ converging weak ${ }^{*}$ to a norm-one element $x^{*}$ then $\lim \left\|x_{n}^{*}-x^{*}\right\|=0$.

Theorem 3.1. Let $X$ be a reflexive Banach space. The following statements are equivalent:

(1) $X^{*}$ is (sequentially) weak ${ }^{*}$ Kadec.

(2) The duality map on $X$ is norm-usco.

(3) If $x \in X /\{0\}$ and $x_{n} \in X$ converge weakly to $x$ then there is $\theta$ in ]0,1] such that $\lim \sup \left\|x-\theta x_{n}\right\|<\|x\|$.

(4) If $x \in X /\{0\}$ and $x_{n} \in X$ converge weakly to $x$ then there is $\theta$ in ]0,1] such that $\liminf \left\|x-\theta x_{n}\right\|<\|x\|$.

(5) If $\left\{C_{n}\right\}$ is a sequence of closed convex subsets of $X$, for which $\lim _{W} C_{n}$ exists and is closed, then $\lim _{M} C_{n}=\lim _{W} C_{n}$.

(6) If $f_{n}$ and $f$ are elements of $X^{*}$ and $\lim _{W} f_{n}^{-1}(1)=f^{-1}(1)$, then $\lim _{M} f_{n}^{-1}(1)=f^{-1}(1)$.

(7) If $x_{n} \in X$ converge weakly to $x$ and $f_{n} \in X^{*}$ converge weak ${ }^{*}$ to $f$ such that $\left\|f_{n}\right\|=\|f\|=\left\langle f_{n}, x_{n}\right\rangle=1$, then $\langle f, x\rangle=1$.

Proof. (1) $\Rightarrow(2)$. Let $\left\{x_{n}\right\}$ converge in norm to $x$ and $f_{n} \in J\left(x_{n}\right)$. Then, as $J$ is locally bounded, there is a subsequence $\left\{f_{n^{\prime}}\right\}$ weakly converging to some $f$. Since

$$
\left\|f_{n^{\prime}}\right\|^{2}=\left\|x_{n^{\prime}}\right\|^{2}=\left\langle f_{n^{\prime}}, x_{n}\right\rangle
$$

while $x_{n^{\prime}} \rightarrow x$ we have

$$
\langle f, x\rangle=\lim \left\langle f_{n^{\prime}}, x\right\rangle=\lim \left\langle f_{n^{\prime}}, x_{n^{\prime}}\right\rangle=\lim \left\|x_{n^{\prime}}\right\|^{2}=\|x\|^{2} .
$$

So $\|f\| \geq \lim \left\|f_{n^{\prime}}\right\|$ and so, by weak lower semicontinuity of the dual norm, $\|f\|=\lim \left\|f_{n^{\prime}}\right\|$. By the Kadec property, $\left\|f_{n^{\prime}}-f\right\| \rightarrow 0$. 
Some consideration shows that this implies (a) that for all $\varepsilon>0$ there is $\delta>0$ with $J(x)+B[0, \varepsilon] \supseteq J(B[x, \delta])$; and (b) that $J(x)$ is norm-compact. $(2) \Rightarrow(3)$. Let $x \neq 0$ and $\left\{x_{n}\right\}$ converge weakly to $x$. Let $\varepsilon>0$ with $\varepsilon \sup \left\|x_{n}\right\|<\|x\|^{2}$. Pick $\theta$ in $\left.] 0,1\right]$ and $f_{n} \in J\left(x-\theta x_{n}\right)$ so that $d\left(f_{n}, J(x)\right)$ $<\varepsilon$ for all $n$, as is possible since $\left\{x_{n}\right\}$ is bounded and $J$ is norm-usco. Choose a subsequence $\left\{x_{n^{\prime}}\right\}$ with $\lim \left\|x-\theta x_{n^{\prime}}\right\|=\lim \sup \left\|x-\theta x_{n}\right\|$. Select $g_{n} \in J(x)$ with $\left\|f_{n}-g_{n}\right\|<\varepsilon$ for all $n$. Since $J(x)$ is compact we may assume that $\left\{g_{n^{\prime}}\right\}$ converges in norm to some $g \in J(x)$. Eventually we have $\left\|f_{n^{\prime}}-g\right\|<\varepsilon$.

We have, from the subgradient property of $J$,

$$
\begin{aligned}
\left\{\left\|x-\theta x_{n^{\prime}}\right\|^{2}-\|x\|^{2}\right\} / 2 & \leq-\left\langle f_{n^{\prime}}, \theta x_{n^{\prime}}\right\rangle \\
& =-\left\langle f_{n^{\prime}}-g, \theta x_{n^{\prime}}\right\rangle-\left\langle g, \theta x_{n^{\prime}}\right\rangle \\
& \leq-\theta\left\langle g, x_{n^{\prime}}\right\rangle+\theta \varepsilon\left\|x_{n^{\prime}}\right\| .
\end{aligned}
$$

Thence

$$
\lim \left\{\left\|x-\theta x_{n^{\prime}}\right\|^{2}-\|x\|^{2}\right\} / 2 \leq \theta\left(\varepsilon \sup \left\|x_{n^{\prime}}\right\|-\|x\|^{2}\right)<0,
$$

which shows that $\|x\|>\lim \left\|x-\theta x_{n^{\prime}}\right\|=\lim \sup \left\|x-\theta x_{n}\right\|$.

(3) $\Rightarrow(4)$. This is immediate.

(4) $\Rightarrow(5)$. Let $C_{n}$ and $C$ be closed convex sets with $d\left(x, C_{n}\right) \rightarrow d(x, C)$ for all $x$. If $C=\varnothing$ then Proposition 2.1(a) shows $\lim _{M} C_{n}=\varnothing$. If $x \in C$ then $d(x, C)=0$ so that $d\left(x, C_{n}\right) \rightarrow 0$ and one can find $c_{n} \in C_{n}$ with $c_{n} \rightarrow x$. Hence $\mathrm{M}(\mathrm{i})$ holds. Now suppose that $x_{n^{\prime}} \in C_{n^{\prime}}$ and $x_{n^{\prime}}$ converges weakly to $x$. We must show that $x \in C$. Otherwise we let $c \neq x$ be the nearest point in $C$ to $x$ ( $C$ is convex and closed, and so proximinal). By translation we arrange that $c=0$, so that $d(x, C)=\|x\|$ and $0 \in C$. As above we can find $c_{n} \in C_{n}$ with $c_{n} \rightarrow 0$. Let $y_{n^{\prime}}:=x_{n^{\prime}}-c_{n^{\prime}}$ which converges weakly to $x \neq 0$.

We apply (4) to $\left\{y_{n^{\prime}}\right\}$ and $x$. For $\theta$ as promised we observe that

$$
\theta y_{n^{\prime}}+c_{n^{\prime}}=\theta\left(x_{n^{\prime}}-c_{n^{\prime}}\right)+c_{n^{\prime}} \in C_{n^{\prime}}
$$

and so

$$
d\left(x, C_{n^{\prime}}\right)-\|x\| \leq\left\|x-\left(\theta y_{n^{\prime}}+c_{n^{\prime}}\right)\right\|-\|x\| \leq\left\|c_{n^{\prime}}\right\|+\left\|x-\theta y_{n^{\prime}}\right\|-\|x\| .
$$

Thus

$$
\begin{aligned}
0=d(x, C)-\|x\| & =\lim d\left(x, C_{n^{\prime}}\right)-\|x\| \leq \liminf \left[\left\|c_{n^{\prime}}\right\|+\left\|x-\theta y_{n^{\prime}}\right\|-\|x\|\right] \\
& =\liminf \left[\left\|x-\theta y_{n^{\prime}}\right\|-\|x\|\right]<0,
\end{aligned}
$$

a clear contradiction. Thus $x \in C$, giving $\mathbf{M}(\mathrm{ii})$.

$(5) \Rightarrow(6)$. Let $C_{n}:=f_{n}^{-1}(1)$ and $C:=f^{-1}(1)$.

$(6) \Rightarrow(7)$. We have $x_{n} \in X$ converging weakly to $x$ and $f_{n} \in X^{*}$ converging weak $^{*}$ to $f$ such that $\left\|f_{n}\right\|=\|f\|=\left\langle f_{n}, x_{n}\right\rangle=1$. Let $C_{n}:=f_{n}^{-1}(1)$ and $C:=f^{-1}(1)$. A computation shows that

$$
d\left(x, C_{n}\right)=\left|1-\left\langle f_{n}, x\right\rangle\right| \text { and } d(x, C)=|1-\langle f, x\rangle|,
$$


so that $d\left(x, C_{n}\right) \rightarrow d(x, C)$ for all $x$. Thus by (6) $\lim _{M} C_{n}=C$. Now $x_{n} \in C_{n}$ and $\left\{x_{n}\right\}$ converges weakly to $x$, so that $x \in C$ and $\langle f, x\rangle=1$.

$(7) \Rightarrow(1)$. We will establish (1) in the form:

$\left(1^{\prime}\right)$ If $\left\|f_{n}\right\|=\|f\|=1$ and $\left\{f_{n}\right\}$ converges weak ${ }^{*}$ to $f$, then $\left\{f_{n}\right\}$ converges to $f$ uniformly on weakly compact sets. Since $X$ is reflexive,

$\left(1^{\prime}\right)$ is equivalent to $(1)$.

Suppose $\left(1^{\prime}\right)$ fails. Then select a weakly compact set $W$ and a subsequence $\left\{f_{n^{\prime}}\right\}$ of $\left\{f_{n}\right\}$ with

$$
\sup _{w \in W}\left|\left\langle f_{n^{\prime}}-f, w\right\rangle\right|>\varepsilon>0 .
$$

Let $x_{n^{\prime}} \in W$ with $\left|\left\langle f_{n^{\prime}}-f, x_{n^{\prime}}\right\rangle\right|>\varepsilon$, and extracting subsequences we may assume that $\left\{x_{n^{\prime}}\right\}$ converges weakly to $x$ and $\left\langle f_{n^{\prime}}, x_{n^{\prime}}\right\rangle \rightarrow \alpha \neq\langle f, x\rangle$. Then

$$
y_{n^{\prime}}:=\frac{x_{n^{\prime}}-x}{\left\langle f_{n^{\prime}}, x_{n^{\prime}}-x\right\rangle} \quad \text { converges weakly to } 0,
$$

and since $\left\langle f_{n^{\prime}}, y_{n^{\prime}}\right\rangle=1=\left\|f_{n^{\prime}}\right\|=\|f\|$ and $\left\{y_{n^{\prime}}\right\}$ converges weakly to 0 , we obtain from $(7)$ that $\langle f, 0\rangle=1$. This contradiction establishes $\left(1^{\prime}\right)$ and completes our circuit.

Remark 3.2. (a) Let us reiterate that these equivalences hold for any finitedimensional normed space [obvious from (3)] or when the norm is Fréchet differentiable [obvious from (2)].

(b) Note in a reflexive space that there is a complete duality between the Kadec condition (1) and the Tsukada conditions (3) and (4).

(c) In each of conditions (3) and (4) " $<$ " may be replaced by " $\leq$ ", as follows if $X_{n}$ is replaced by $2 x_{n}-x$ in the nonstrict version.

Corollary 3.3. If $X$ is a smooth reflexive normed space then the norm on $X$ is Fréchet if and only if the equivalences (1) through (7) of Theorem 3.1 hold.

Proof. Since $X$ is reflexive, the norm is Fréchet precisely when the dual norm is strictly convex and $\mathrm{Kadec}$, or equivalently when the original norm is smooth and the dual norm is Kadec [Ho, Ts, Bo-Fi].

Theorem 3.4. A Banach space is reflexive and dual Kadec if and only if Mosco and Wijsman convergences coincide for sequences of closed convex sets.

Proof. Combine the equivalence of (1) and (5) of Theorem 3.1 with Corollary 2.3.

The next result extends and unifies Theorem 3.3 of Tsukada [Ts].

Corollary 3.5. If the norm on $X^{*}$ is Fréchet differentiable (so $X$ is reflexive) and Kadec, then the following are equivalent for any sequence $\left\{C_{n}\right\}$ of closed nonempty convex subsets of $X$.

(1) $\lim _{M} C_{n}$ exists and is nonempty.

(2) $\lim _{W} C_{n}$ exists and is nonempty.

(3) For every $x \in X$ the sequence of metric projections of $x$ onto $C_{n}$ is norm convergent. 
Proof. This follows by Tsukada's arguments [Ts, pp. 306-307] on replacing his Theorem 2.5 by our Theorem 3.4.

We note that one can explicitly give norms on $l_{2}$ which are smooth but for which the dual is not Kadec: let \|\| be the Hilbert norm on $l_{2}$ and set $\|\mid(r, x)\|\left\|^{2}:=\right\|(r, T x) \|^{2}+\max \{\|x\|,|r|\}^{2}$ where $(T x)_{n}:=x_{n} / n$. Then the dual norm is smooth but its dual is not Kadec. So we have a smooth equivalent norm on Hilbert space for which Mosco and Wijsman convergences do not coincide for sequences of closed convex sets.

We finish this section by noting a remarkable duality.

Theorem 3.6. Mosco and Wijsman convergences coincide for sequences of closed convex sets in a Banach space $X$ if and only if every closed nonempty subset $C$ of $X^{*}$ is almost proximinal (i.e. there is a generic set of points in $X^{*} \backslash C$ which admit nearest points in $C$ ).

Proof. By the theorem of Lau [La] and Konjagin [Ko], also derived in [Bo-Fi, Theorem 5.11], the second condition also coincides, with $X^{*}$ being reflexive and Kadec.

\section{THE NONREFLEXIVE CASE}

While the results of $\S 2$ show that many things fail in the absence of reflexivity, there is an adequate analogue for Theorem 3.1.

Theorem 4.1. Let $X$ be a Banach space whose dual unit ball is weak ${ }^{*}$ sequentially compact. The following statements are equivalent.

(1) If $f_{n}$ and $f$ are elements of $X^{*}$ with $\left\|f_{n}\right\|=\|f\|=1$ and if $\left\{f_{n}\right\}$ converges weak ${ }^{*}$ to $f$ while $f$ is norm-attaining, then $\left\{f_{n}\right\}$ converges to $f$ uniformly on weakly compact sets, that is to say in the Mackey topology $\tau:=\tau\left(X^{*}, X\right)$.

(2) The duality map on $X$ is norm- $\tau$ upper semicontinuous with sequentially $\tau$-compact images.

(3) If $x \in X /\{0\}$ and $x_{n} \in X$ converge weakly to $x$ then there is $\theta$ in ]0,1] such that lim sup $\left\|x-\theta x_{n}\right\|<\|x\|$.

(4) If $x \in X /\{0\}$ and $x_{n} \in X$ converge weakly to $x$ then there is $\theta$ in ]0,1] such that $\liminf \left\|x-\theta x_{n}\right\|<\|x\|$.

(5) If $\left\{C_{n}\right\}$ is a sequence of closed convex subsets of $X$, for which $\lim _{W} C_{n}$ exists and is proximinal, then $\lim _{M} C_{n}=\lim _{W} C_{n}$.

(6) If $f_{n}$ and $f$ are elements of $X^{*}$ with $f$ norm-attaining and $\lim _{W} f_{n}^{-1}(1)$ $=f^{-1}(1)$, then $\lim _{M} f_{n}^{-1}(1)=f^{-1}(1)$.

(7) If $x_{n} \in X$ converge weakly to $x$ and $f_{n} \in X^{*}$ converge weak ${ }^{*}$ to a norm-attaining element $f \in X^{*}$ such that $\left\|f_{n}\right\|=\|f\|=\left\langle f_{n}, x_{n}\right\rangle=1$, then $\langle f, x\rangle=1$.

Proof. The proof proceeds essentially as in Theorem 3.1, replacing the norm topology on $X^{*}$ by the Mackey topology $\tau$ (see [GGS]). 
For any Banach space with an equivalent smooth norm the dual unit ball is weak * sequentially compact. This holds whenever the space is WCG and so if $X$ is separable or reflexive.

Corollary 4.2. Let $X$ be a Banach space whose dual unit ball is weak ${ }^{*}$ sequentially compact. Supposes that either (1) the dual norm is sequentially weak ${ }^{*}$ Kadec or (2) the norm is Fréchet differentiable. If $\left\{C_{n}\right\}$ is a sequence of closed convex subsets of $X$, and $\lim _{W} C_{n}$ exists and is proximinal, then $\lim _{M} C_{n}=\lim _{W} C_{n}$.

Proof. (1) and (2) are stronger than (1) and (2) of Theorem 4.1.

Example 4.3. (a) Let $X$ be $c_{0}(S)$ for any set $S$, endowed with the supremum norm. Then Corollary 4.2(1) holds.

(b) Let $X$ be $c_{0}(S)$, endowed with any Fréchet differentiable renorm. Then Corollary $4.2(2)$ holds.

\section{ACKNOWLEDGMENT}

We would like to thank John Giles and the Department of Mathematics at the University of Newcastle, Australia, and the Centre for Mathematical Analysis at the Australian National University for their support and hospitality while this work was in progress. We would like to thank John Giles and Gar de Barra for many useful conversations about this work.

\section{REFERENCES}

[At] H. Attouch, Variational convergence for functions and operators, Pitman, Boston, 1984.

[Au] J. P. Aubin, Applied abstract analysis, Wiley, New York, 1977.

[Be] G. Beer, Convergence of continuous linear functionals and their level sets, Arch. Math. (in press).

[Bo-Fi] J. M. Borwein and S. P. Fitzpatrick, Existence of nearest points in Banach spaces, Canadian Journal of Mathematics (in press).

[Da] M. M. Day, Normed linear spaces, third edition, Springer-Verlag, New York, 1973.

[GGS] J. R. Giles, D. A. Gregory and B. Sims, Geometrical implications of upper semicontinuity of the duality mapping on a Banach space, Pacific J. Math. 79 (1978), 99-109.

[Ho] R. B. Holmes, A course on optimization and best approximation, Lecture Notes in Mathematics, no. 257, Springer-Verlag, New York, 1972.

[Ko] S. V. Konjagin, On approximation properties of closed sets in Banach spaces and the characterization of strongly convex spaces, Soviet Math. Dokl. 21 (1980), 418-422.

[La] K.-S. Lau, Almost Chebychev subsets in reflexive Banach spaces, Indiana Univ. Math. J. 27 (1978), 791-795.

[Mo1] U. Mosco, Convergence of convex sets and of solutions of variational inequalities, Adv. in Math. 3 (1969), 510-585.

[Mo2] _ _ "On the continuity of the Young-Fenchel transform,” J. Math Anal. Appl. 35 (1971), 518-535.

[Sa-We] G. Salinetti and R. Wets, Convergence of convex sets in finite dimensions, SIAM Rev. 21 (1979), 18-33.

[So] Y. Sonntag, Convergence au sens de Mosco; théorie et applications à l'approximation des solutions d'inéquations, Thèse d’Ėtat. Université de Provence, Marseille, 1982. 
[Ts] M. Tsukada, Convergence of best approximations in a smooth Banach space, J. Approx. Theory 40 (1984), 301-309.

[Wi] R. Wijsman, Convergence of sequences of convex sets, cones, and functions, II, Trans. Amer. Math. Soc. 123 (1966), 32-45.

Department of Mathematics, Statistics and Computer Science, Dalhousie University, Halifax, Nova Scotia, Canada B3H $3 J 5$

Department of Mathematics and Statistics, University of Auckland, Private bag, Auckland, New Zealand 